01

\title{
Влияние изотопозамещения на параметры разложения эффективного дипольного момента в молекулах типа $\mathrm{XY} / \mathrm{XYZ}$
}

\author{
(C) А.Г. Зятькова, А.С. Белова, О.В. Громова, Е.С. Бехтерева \\ Национальный исследовательский Томский политехнический университет, \\ 634050 Томск, Россия \\ e-mail: anastasia.litvinovskaya@mail.ru
}

Поступила в редакцию 06.02.2019 г.

В окончательной редакции 13.05.2019 г.

Принята к публикации 16.05.2019 г.

\begin{abstract}
Обсуждены особенности изменения дипольных характеристик молекул при изотопическом замещении. Для случая несимметричного замещения $\mathrm{XYZ} \leftarrow \mathrm{XY}_{2}$ на основе использования операторной теории возмущений и теории групп впервые получены аналитические выражения для шести параметров разложения эффективного дипольного момента $\left({ }^{a_{i}} \mu_{1},{ }^{a_{i}} \mu_{4},{ }^{a_{i}} \mu_{5},{ }^{a_{i}} \mu_{9},{ }^{a_{i}} \mu_{12}\right.$ и $\left.{ }^{a_{i}} \mu_{14}\right)$. Получены соотношения, позволяющие связать между собой равновесный дипольный момент и первые производные дипольного момента „материнской“ и изотопозамещенной молекул. На примере молекул ${ }^{32} \mathrm{~S}^{16} \mathrm{O}_{2},{ }^{34} \mathrm{~S}^{16} \mathrm{O}_{2},{ }^{32} \mathrm{~S}^{18} \mathrm{O}_{2}$ и ${ }^{32} \mathrm{~S}^{16} \mathrm{O}^{18} \mathrm{O}$ выполнен количественный расчет параметров эффективного дипольного момента.
\end{abstract}

Ключевые слова: изотопозамещение, эффективный дипольный момент, теория возмущений.

DOI: $10.21883 /$ OS.2019.09.48187.48-19

\section{1. Введение}

В настоящее время одной из наиболее актуальных проблем молекулярной спектроскопии является исследование интенсивностей колебательно-вращательных линий. С прикладной точки зрения важность указанной проблемы определяется тем, что на основе информации об интенсивностях линий, а также данных, полученных путем дистанционного зондирования, можно количественно определять характеристики среды, такие как температура, давление, концентрация и т.д. Такой подход чрезвычайно важен в астрофизике при исследовании атмосфер планет, межзвездной среды, в атмосферной оптике при исследовании озонового слоя, контроле загрязнений, анализе парниковых газов, свойств прозрачности атмосферы Земли [1,2].

Известно [3], что в отсутствие внешнего поля интенсивность спектральной линии равна интегралу от коэффициента поглощения $\alpha(\sigma)$ :

$$
\begin{aligned}
S_{\sigma}^{N}= & \int \alpha\left(\sigma^{\prime}\right) d \sigma^{\prime}=\frac{8 \pi^{3} \sigma}{4 \pi \varepsilon_{0} 3 h c}\left(1-\exp \left(-\frac{h c \sigma}{k T}\right)\right) \\
& \times N \frac{g_{A}}{Z(T)} \exp \left(-\frac{E_{A}}{k T}\right) R_{A}^{B},
\end{aligned}
$$

где $E_{A}$ и $E_{B}$ определяют верхнее и нижнее состояния перехода, $\sigma=\left(E_{B}-E_{A}\right) / h c-$ волновое число соответствующего колебательного перехода, $g_{A}$ - статистический вес нижнего состояния $A, Z(T)$ - статистическая сумма, зависящая от температуры, $N$ - количество поглощающих молекул в единице объема. Величина $R_{A}^{B}$ определяет квадрат матричного элемента дипольного момента на собственных колебательно-вращательных функциях свободной молекулы:

$$
R_{A}^{B}=\sum_{a} \sum_{b} 3\left|\left\langle a\left|P_{\alpha}\right| b\right\rangle\right|^{2},
$$

здесь $a$ и $b$ представляют состояния, соответствующие уровням $A$ и $B$, имеющим в отсутствие внешнего поля $M$ вырождение.

Все параметры, кроме $R_{A}^{B}$, входящие в уравнение (1), являются известными или их расчет не вызывает затруднений. Задача определения величины $R_{A}^{B}$ до сих пор относится к нетривиальным задачам. В связи с этим основная проблема при исследовании интенсивностей линий поглощения заключается в расчете матричных элементов $\left\langle a\left|P_{\alpha}\right| b\right\rangle$ оператора дипольного момента. В работах $[3,4]$ предложен подход для определения интенсивности конкретной линии в спектрах молекул типа $\mathrm{XY}_{2}$ на основе теории эффективного дипольного момента. Параметры в разложении эффективного дипольного момента позволяют оценивать значения интенсивностей линий и предсказывать интенсивность „проблемных“ линий (например, накрытых, перенасыщенных, слабых и т.д.). Однако для случаев, когда не хватает экспериментальной информации о колебательных состояниях (например, для ранее не исследованных или плохо изученных изотопологов) упомянутый подход является проблематичным. В этом случае незаменимым инструментом выступает теория изотопозамещения [5-8], которая позволяет определять параметры изотополога как функции хорошо изученных фундаментальных постоянных „материнской“ молекулы.

В настоящей работе влияние изотопозамещения на параметры разложения эффективного дипольного момента в молекулах типа $\mathrm{XY}_{2} / \mathrm{XYZ}$ рассматривается на примере молекулы $\mathrm{SO}_{2}$ и ее изотопологов ${ }^{32} \mathrm{~S}^{16} \mathrm{O}_{2},{ }^{34} \mathrm{~S}^{16} \mathrm{O}_{2}$, 
${ }^{32} \mathrm{~S}^{18} \mathrm{O}_{2}$ и ${ }^{32} \mathrm{~S}^{16} \mathrm{O}^{18} \mathrm{O}$. В общем случае симметрия этих молекул при одновременном замещении ядер кислорода либо при замещении ядра серы не изменяется. Для данного класса молекул в работе [4] получены формулы для параметров в разложении эффективного дипольного момента. Однако в случае замещения одного ядра кислорода молекулы ${ }^{32} \mathrm{~S}^{16} \mathrm{O}^{18} \mathrm{O}$ происходит смещение и поворот системы координат, приводящие к понижению симметрии изотополога $\left(C_{s} \leftarrow C_{2 v}\right)$. Для такого сорта молекул формулы, описывающие параметры в разложении эффективного дипольного момента, отсутствуют в литературе на сегодняшний день. Для исследования влияния изотопозамещения параметры в разложении эффективного дипольного момента молекул типа XYZ $\left(C_{s}\right.$ симметрии) были получены в аналитическом виде.

\section{2. Элементы операторной теории возмущений}

Как отмечалось выше, основная проблема при теоретическом исследовании интенсивностей линий поглощения заключается в расчете матричного элемента дипольного момента на собственных колебательно-вращательных функциях гамильтониана [9-12]. Однако решение уравнения Шредингера, т.е. однозначное определение колебательно-вращательных собственных функций и энергий, возможно только для простых молекулярных систем. В связи с этим определение собственных функций, необходимых для расчета интенсивностей спектральных линий, является трудновыполнимой задачей. По этой причине, начиная с 30-х годов прошлого века, уделяется огромное внимание разработке альтернативных методов решения упомянутой проблемы. В подавляющем большинстве случаев для решения задачи используют различные разновидности так называемого метода эффективных операторов, например, метод контактных преобразований, метод эффективного нежесткого волчка, операторная проекционная формулировка и т.д.

В качестве одного из вышеперечисленных методов в настоящей работе используется метод эффективных операторов в рамках операторной теории возмущений. Рассмотрим подробнее процесс построения и расчета матричного элемента $\left\langle a\left|P_{\alpha}\right| b\right\rangle$ в рамках выбранного метода.

Отметим, что в дальнейшем будем рассматривать колебательно-вращательную структуру выделенного электронного состояния. В этом случае будем говорить, что $P_{\alpha}^{\gamma \beta}-$ усредненный оператор дипольного момента для данного электронного состояния:

$$
P_{\alpha}^{\gamma \beta}=\left\langle\psi_{\gamma}\left|P_{\alpha}\right| \psi_{\beta}\right\rangle .
$$

Здесь $\psi_{\gamma}, \psi_{\beta}$ являются собственными функциями колебательно-вращательного гамильтониана

$$
H=H_{0}(t)+h(t, k),
$$

где $H_{0}$ - оператор, зависящий только от колебательных координат (гармоническое приближение), $h(t, k)-$ оператор, учитывающий зависимость гамильтониана как от колебательных, так и от вращательных переменных. Перейдем от оператора (3) к эквивалентному ему оператору, выполнив унитарное преобразование:

$$
\widetilde{H}=G^{+} H G \text {. }
$$

Тогда собственные волновые функции оператора $H$ примут вид

$$
\left|\psi_{\gamma}\right\rangle=\sum_{a, b} G_{a b}^{\gamma}|a b\rangle
$$

Здесь $G_{a b}^{\gamma}$ - элементы унитарной матрицы $G$, приводящей к диагональному исходную матрицу $H,|a\rangle-$ полный ортонормированный набор функций, зависящий от колебательных переменных, $|b\rangle-$ совокупность ортонормированных функций, зависящих от вращательных переменных, удовлетворяющих условию полноты. Тогда $|a b\rangle=|a\rangle|b\rangle$ также будут образовывать полный ортонормированный набор функций в гильбертовом пространстве, в котором действует оператор $H$.

С учетом (4) уравнение (2) можно записать как

$$
\left\langle a\left|\left\langle b\left|G^{+} P_{\alpha} G\right| b^{\prime}\right\rangle\right| a^{\prime}\right\rangle=\left\langle a\left|\left\langle b\left|\widetilde{P}_{\alpha}\right| b^{\prime}\right\rangle\right| a^{\prime}\right\rangle,
$$

где $\widetilde{P}_{\alpha}-$ преобразованный эффективный дипольный момент. Здесь оператор дипольного момента $P_{\alpha}$ имеет [10] вид

$$
P_{\alpha}=\sum_{\alpha} k_{\alpha \beta}\left(\mu_{\beta}^{e}+\sum_{\lambda} \mu_{\beta}^{\lambda} q_{\lambda}+\sum_{\lambda \theta} \mu_{\beta}^{\lambda \theta} q_{\lambda} q_{\theta}+\ldots\right) .
$$

В выражении (6) коэффициенты $\mu_{\beta}^{e}, \mu_{\beta}^{\lambda}$ и $\mu_{\beta}^{\lambda \theta}, \ldots$ - параметры дипольного момента для данного электронного состояния. На практике данные параметры определяют исходя из экспериментальных данных, $k_{\alpha \beta}-$ элементы матрицы направляющих косинусов.

Для расчета матричных элементов (5) необходимо иметь явный вид элементов $G_{t \beta}^{\gamma}$. Как было показано в работе [13], оператор $G$ удобно выбрать в виде

$$
G=\exp \left(i \sum_{n=1}^{\infty} g_{n}\left(\kappa^{n}\right)\right),
$$

где $g_{n}=g_{n}(t, k)$ - „малые“ эрмитовы операторы порядка $\kappa^{n}$. Разобьем пространство $K_{a}$ волновых функций $|a\rangle$ на два конечномерных подпространства $K_{a_{0}}, K_{a^{\prime}}$ и одно бесконечномерное $K_{a^{\prime \prime}}$, так что $K_{a}=K_{a_{0}}+K_{a^{\prime}}+K_{a^{\prime \prime}}$. Причем $K_{a_{0}}-$ множество состояний, с которых происходит переход, а подпространство $K_{a^{\prime}}$ содержит в себе совокупность резонирующих друг с другом колебательных состояний, на которые происходит переход. Все остальные состояния принадлежат подпространству $K_{a^{\prime \prime}}$. Тогда элементы матрицы $G$ в общем виде определяются 
как

$$
\begin{gathered}
\left\langle\alpha\left|i g_{1}\right| \beta\right\rangle=\frac{h_{\alpha \beta}}{E_{\beta}-E_{\alpha}} \\
\left\langle\alpha\left|i g_{2}\right| \beta\right\rangle=\frac{1}{2} \sum_{\gamma \notin \alpha, \beta} \frac{h_{\alpha \gamma} h_{\gamma \beta}}{E_{\beta}-E_{\alpha}}\left(\frac{1}{E_{\beta}-E_{\gamma}}+\frac{1}{E_{\alpha}-E_{\gamma}}\right) \\
+\frac{\left(h_{\alpha \alpha} h_{\alpha \beta}-h_{\alpha \beta} h_{\beta \beta}\right)}{\left(E_{\beta}-E_{\alpha}\right)^{2}}
\end{gathered}
$$

и т.Д.

Здесь $|\alpha\rangle$ и $|\beta\rangle$ - колебательные волновые функции, принадлежащие разным подпространствам. Также введено обозначение $h_{\alpha \beta}=\langle\alpha|h| \beta\rangle$. Вид оператора $h$ взят из работы [14].

\section{3. Определение параметров эффективного дипольного момента молекулы типа XYZ симметрии $C_{s}$}

Молекула ${ }^{32} \mathrm{~S}^{16} \mathrm{O}^{18} \mathrm{O}$ является молекулой типа асимметричного волчка, группа симметрии которой изоморфна точечной группе $C_{s}$. Выберем оси молекулярно фиксированной системы координат в соответствии с $I^{r}$-представлением А-редуцированного эффективного гамильтониана типа Уотсона (рисунок). Как было показано в работе [3], преобразованный эффективный дипольный момент может быть представлен в следующей форме:

$$
\widetilde{P}_{\alpha}=\sum_{\beta} \sum_{i}\left\{O_{i}, k_{\alpha \beta}\right\}
$$

где $O_{i}$ - колебательные операторы, $\{A, B\}=A B+B A$. Оператор дипольного момента $P_{\alpha}$ в случае симметрии $C_{s}$ должен преобразовываться по представлению $A^{\prime \prime}$. В этом случае правая часть уравнения (8) должна содержать тип симметрии $A^{\prime \prime}$. Если представить оператор как $O_{i}=O_{i}^{\mathrm{V}} O_{i}^{\mathrm{R}}$, тогда прямое произведение типов симметрии колебательного и вращательного операторов будет

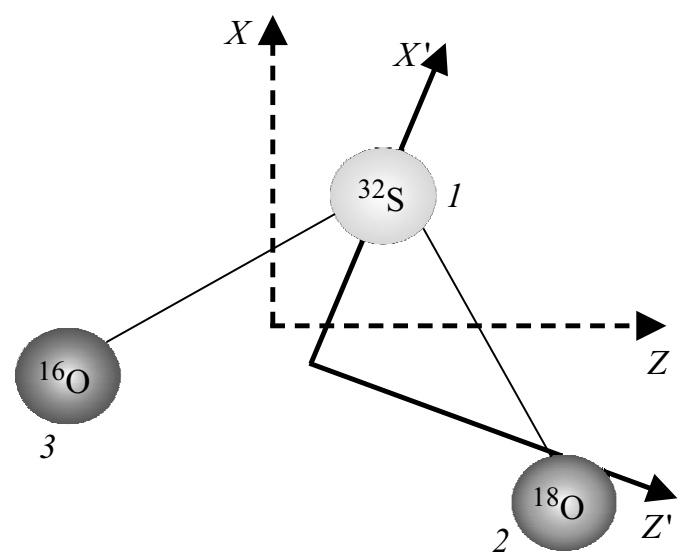

Определение осей молекулы ${ }^{32} \mathrm{~S}^{16} \mathrm{O}^{18} \mathrm{O} . X, Z-$ оси, соответствующие основной конфигурации $\left(\mathrm{SO}_{2}\right) ; X^{\prime}, Z^{\prime}-$ оси координатной системы молекулы ${ }^{32} \mathrm{~S}^{16} \mathrm{O}^{18} \mathrm{O}$.
Таблица 1. Типы симметрии и неприводимые представления точечной группы $C_{s}$

\begin{tabular}{c|rr|ccc}
\hline$C_{s}$ & $E$ & $\sigma_{h}$ & \multicolumn{3}{|c}{ Базисы } \\
\hline$A^{\prime}$ & 1 & 1 & $k_{z y}$ & $J_{y}$ & $q_{1}, q_{2}, q_{3}$ \\
$A^{\prime \prime}$ & 1 & -1 & $k_{z x}, k_{z z}$ & $J_{x}, J_{z}$ &
\end{tabular}

Таблица 2. Типы симмметрии операторов $O_{i}=O_{i}^{\mathrm{V}} O_{i}^{\mathrm{R}}$

\begin{tabular}{c|c|c|c|c}
\hline$\beta$ & $\Gamma\left(k_{z \beta}\right)$ & $\Gamma\left(O_{i}^{\mathrm{V}}\right)$ & $\Gamma\left(O_{i}^{\mathrm{R}}\right)$ & $O_{i}^{\mathrm{R}}$ \\
\hline$x$ & $A^{\prime \prime}$ & & $A^{\prime}$ & $\begin{array}{c}1, J^{2}, J_{z}^{2}, J_{x y}^{2} \\
\left(J_{x} J_{z}+J_{z} J_{x}\right), J_{y}\end{array}$ \\
\cline { 1 - 2 }$y$ & $A^{\prime}$ & $A^{\prime}$ & & $\begin{array}{l}\left(J_{x} J_{y}+J_{y} J_{x}\right), J_{z} \\
\left(J_{y} J_{z}+J_{z} J_{y}\right), J_{x}\end{array}$
\end{tabular}

определяться симметрией операторов $q_{1}, q_{2}, q_{3}$ и $J_{x}$, $J_{y}, J_{z}$ соответственно. Неприводимые представления и таблица характеров группы симметрии $C_{s}$ представлены в табл. 1 [10].

Все возможные комбинации для операторов $O_{i}^{\mathrm{V}}$ и $O_{i}^{\mathrm{R}}$ даны в табл. 2 [10]. Вместе с тем можно показать, учитывая уравнение (5), что оператор эффективного дипольного момента для конкретного колебательного состояния (переход происходит с основного состояния на колебательное состояние $a_{i}$ ) примет вид

$$
{ }^{a_{i}} \mu_{\alpha}=\sum_{i}\left\langle 0\left|\widetilde{P}_{\alpha}\right| a_{i}\right\rangle
$$

где $\left|a_{0}\right\rangle=|000\rangle,\left|a_{1}\right\rangle=|100\rangle,\left|a_{2}\right\rangle=|010\rangle$ и $\left|a_{3}\right\rangle=|001\rangle$. С другой стороны, можно показать [3], что оператор эффективного дипольного момента ${ }^{a_{i}} \mu_{\alpha}$ может быть представлен в виде выражения

$$
{ }^{a_{i}} \mu_{\alpha}=\sum_{j}{ }^{a_{i}} \mu_{j}{ }^{a_{i}} A_{j} .
$$

Здесь ${ }^{a_{i}} \mu_{j}$ - численные коэффициенты и ${ }^{a_{i}} A_{j}-$ симметризованные операторы.

На основании табл. 2, а также применяя основные принципы операторной теории возмущений (разд. 2), можно показать, что оператор эффективного дипольного момента молекул, обладающих симметрией $C_{s}$, имеет вид алгебраической суммы 16 операторов, перечисленных в табл. 3. Отличие от нуля операторов $k_{z x}$ и $k_{z z}$ приводит к тому, что в колебательно-вращательных спектрах молекул такого типа одновременно наблюдаются переходы типов как $a$, так и $b$, т. е. все полосы, активные в инфракрасном диапазоне, являются гибридными.

В качестве основы определения формул для параметров эффективного дипольного момента используем преобразования, описанные в разд. 2. Запишем в явном 
Таблица 3. Симметризованные операторы, характеризующие эффективный дипольный момент молекул типа XYZ (симметрии $C_{s}$ )

\begin{tabular}{c|c|c|c}
\hline$j$ & ${ }^{a_{i}} A_{j}$ & $j$ & ${ }^{a_{i}} A_{j}$ \\
\hline 1 & $k_{z x}$ & 9 & $k_{z z}$ \\
\hline 2 & $\left\{k_{z x}, J^{2}\right\}$ & 10 & $\left\{k_{z z}, J^{2}\right\}$ \\
\hline 3 & $\left\{k_{z x}, J_{z}^{2}\right\}$ & 11 & $\left\{k_{z z}, J_{z}^{2}\right\}$ \\
\hline 4 & $\left\{i k_{z y}, J_{z}\right\}$ & 12 & $\frac{1}{2}\left[\left\{k_{z x}, i J_{y}\right\}-\left\{i k_{z y}, J_{x}\right\}\right]$ \\
\hline 5 & $\left\{k_{z z}, i J_{y}\right\}$ & 13 & $\frac{1}{2}\left[\left\{k_{z x}, J_{x} J_{z}+J_{z} J_{x}\right\}-\left\{i k_{z y}, i\left(J_{y} J_{z}+J_{z} J_{y}\right)\right\}\right]$ \\
\hline 6 & $\left\{k_{z z}, J_{x} J_{z}+J_{z} J_{x}\right\}$ & 14 & $\frac{1}{2}\left[\left\{k_{z x}, i J_{y}\right\}+\left\{i k_{z y}, J_{x}\right\}\right]$ \\
\hline 7 & $\frac{1}{2}\left[\left\{k_{z x}, J_{x y}^{2}\right\}-\left\{i k_{z y}, i\left(J_{x} J_{y}+J_{y} J_{x}\right)\right\}\right]$ & 15 & $\frac{1}{2}\left[\left\{k_{z x}, J_{x} J_{z}+J_{z} J_{x}\right\}+\left\{i k_{z y}, i\left(J_{y} J_{z}+J_{z} J_{y}\right)\right\}\right]$ \\
\hline 8 & $\frac{1}{2}\left[\left\{k_{z x}, J_{x y}^{2}\right\}+\left\{i k_{z y}, i\left(J_{x} J_{y}+J_{y} J_{x}\right)\right\}\right]$ & 16 & $\left\{k_{z z}, J_{x y}^{2}\right\}$ \\
\hline
\end{tabular}

виде уравнение (5) с учетом (7):

$$
\begin{aligned}
& \widetilde{P}_{z}=\langle a|\left(1-i \sum_{n=1} g_{n}\left(\kappa^{n}\right)+\frac{1}{2}\left(i \sum_{n=1} g_{n}\left(\kappa^{n}\right)\right)^{2}-\ldots\right) \\
& \times\left(\sum_{\alpha} k_{z \alpha}\left\{\mu_{\alpha}^{e}\left(\kappa^{0}\right)+\sum_{\lambda} \mu_{\alpha}^{\lambda} q_{\lambda}\left(\kappa^{1}\right)+\sum_{\lambda \leq v} \mu_{\alpha}^{\lambda v} q_{\lambda} q_{v}\left(\kappa^{2}\right) \ldots\right\}\right) \\
& \times\left(1+i \sum_{n=1} g_{n}\left(\kappa^{n}\right)+\frac{1}{2}\left(i \sum_{n=1} g_{n}\left(\kappa^{n}\right)\right)^{2}+\ldots\right)\left|a^{\prime}\right\rangle .
\end{aligned}
$$

Как видно из уравнения (10), результат представляет собой алгебраическую сумму величин различного порядка малости. Раскрыв скобки в уравнении и объединив при этом слагаемые по порядкам малости, можно выделить главные вклады в коэффициенты перед определенными операторами из табл. 3. В процессе анализа было использовано коммутационное соотношение

$$
\left[k_{z i}, J_{j}\right]=-i \varepsilon_{i j k} k_{z k} .
$$

В рамках настоящей работы были получены в явном виде коэффициенты ${ }^{a_{i}} \mu_{j}$ для $1,4,5,9,12$ и 14 параметров в разложении эффективного дипольного момента в соответствии с введенной в табл. 3 нумерацией для фундаментальных полос $v_{1}, v_{2}$ и $v_{3}$ :

$$
\begin{gathered}
{ }^{a_{i}} \mu_{1}=\frac{\mu_{x}^{\lambda}}{\sqrt{2}}, \\
{ }^{a_{i}} \mu_{9}=\frac{\mu_{z}^{\lambda}}{\sqrt{2}}, \\
{ }^{a_{i}} \mu_{4}=\frac{\mu_{x}^{e} \mu_{z z}^{\lambda}}{\sqrt{2} \omega_{\lambda}}-\frac{\mu_{z}^{e}\left(\mu_{x z}^{\lambda}+\mu_{z x}^{\lambda}\right)}{2 \sqrt{2} \omega_{\lambda}},
\end{gathered}
$$

$$
\begin{aligned}
& { }^{a_{i}} \mu_{5}=-\frac{\mu_{x}^{e} \mu_{y y}^{\lambda}}{\sqrt{2} \omega_{\lambda}} \\
& +\left\{\begin{array}{l}
\sum_{k \neq \lambda} \sqrt{2} \mu_{z}^{k} \xi_{\lambda k}^{y} \mu_{y y}^{e} \frac{\sqrt{\omega_{\lambda} \omega_{k}}}{\omega_{k}^{2}-\omega_{\lambda}^{2}}, \\
\sum_{k \neq \lambda} \frac{\sqrt{2}}{4} \frac{\mu_{z}^{k} \xi_{\lambda \mu^{y}}^{e}}{\sqrt{\omega_{\lambda} \omega_{k}}}\left(\frac{\omega_{\lambda}-\omega_{k}}{\omega_{\lambda}+\omega_{k}}+\right. \\
\left.+\frac{\omega_{\lambda}+\omega_{k}}{\omega_{\lambda}-\omega_{k}}\left(1-\delta_{1 k} \delta_{3 \lambda}\right)\left(1-\delta_{3 k} \delta_{1 \lambda}\right)\right),
\end{array}\right\} \\
& { }^{a_{i}} \mu_{12}=\frac{\mu_{z}^{e} \mu_{x x}^{\lambda}}{\sqrt{2} \omega_{\lambda}}+\frac{\mu_{z}^{e} \mu_{y y}^{\lambda}}{\sqrt{2} \omega_{\lambda}}-\frac{\mu_{x}^{e}\left(\mu_{x z}^{\lambda}+\mu_{z x}^{\lambda}\right)}{2 \sqrt{2} \omega_{\lambda}} \\
& +\left\{\begin{array}{l}
\sum_{k \neq \lambda} \sqrt{2} \mu_{x}^{k} \xi_{\lambda k}^{y} \mu_{y y}^{e} \frac{\sqrt{\omega_{\lambda} \omega_{k}}}{\omega_{k}^{2}-\omega_{\lambda}^{2}}, \\
\sum_{k \neq \lambda} \frac{\sqrt{2}}{4} \frac{\mu_{x}^{k} \xi_{\lambda l}^{y} \mu_{y y}^{e}}{\sqrt{\omega_{\lambda} \omega_{k}}}\left(\frac{\omega_{\lambda}-\omega_{k}}{\omega_{\lambda}+\omega_{k}}\right. \\
\left.+\frac{\omega_{\lambda}+\omega_{k}}{\omega_{\lambda}-\omega_{k}}\left(1-\delta_{1 k} \delta_{3 \lambda}\right)\left(1-\delta_{3 k} \delta_{1 \lambda}\right)\right),
\end{array}\right\} \\
& { }^{a_{i}} \mu_{14}=-\frac{\mu_{z}^{e} \mu_{x x}^{\lambda}}{\sqrt{2} \omega_{\lambda}}+\frac{\mu_{z}^{e} \mu_{y y}^{\lambda}}{\sqrt{2} \omega_{\lambda}}+\frac{\mu_{x}^{e}\left(\mu_{x z}^{\lambda}+\mu_{z x}^{\lambda}\right)}{2 \sqrt{2} \omega_{\lambda}} \\
& +\left\{\begin{array}{l}
\sum_{k \neq \lambda} \sqrt{2} \mu_{x}^{k} \xi_{\lambda k}^{y} \mu_{y y}^{e} \frac{\sqrt{\omega_{\lambda} \omega_{k}}}{\omega_{k}^{2}-\omega_{\lambda}^{2}}, \\
\sum_{k \neq \lambda} \frac{\sqrt{2}}{4} \frac{\mu_{x}^{k} \xi_{\lambda k}^{y} \mu_{y y}^{e}}{\sqrt{\omega_{\lambda} \omega_{k}}}\left(\frac{\omega_{\lambda}-\omega_{k}}{\omega_{\lambda}+\omega_{k}}\right. \\
\left.+\frac{\omega_{\lambda}+\omega_{k}}{\omega_{\lambda}-\omega_{k}}\left(1-\delta_{1 k} \delta_{3 \lambda}\right)\left(1-\delta_{3 k} \delta_{1 \lambda}\right)\right) .
\end{array}\right\}
\end{aligned}
$$

Как видно из полученных выражений, данные параметры являются функциями вращательных коэффициентов, гармонических частот, равновесного и первых производных дипольного момента. Данные величины, кроме параметров дипольного момента, рассчитываются с помощью известных в колебательно-вращательной теории формул [14]. Методика расчета параметров дипольного момента будет обсуждена ниже.

Для молекул симметрии $C_{s}$ все колебания преобразуются по неприводимому представлению $A^{\prime}$ (табл. 1). По этой причине между различными состояниями будут одновременно возникать два типа резонанса: резонанс Ферми и резонанс Кориолиса $C_{y}$-типа). В процессе 
получения формул было рассмотрено два случая: а) если учет резонанса не обязателен в рамках рассматриваемой задачи, то необходимо воспользоваться в формулах (14)-(16) верхней строчкой в фигурных скобках; б) принимая в расчет резонансные взаимодействия, в формулах (14)-(16) нижняя часть в фигурных скобках должна быть использована при вычислениях.

\section{4. Изотопические соотношения для параметров дипольного момента симметрично и несимметрично замещенных изотопологов диоксида серы}

В общем случае коэффициенты $\mu_{\alpha}^{e}, \mu_{\alpha}^{\lambda}$ могут быть записаны в виде функций от интегралов по электронным переменным. Поскольку корректное вычисление такого рода интегралов для произвольных многоатомных молекул до сих пор остается трудноразрешимой задачей, на практике эти параметры определяют исходя из экспериментальных данных по интенсивностям колебательновращательных линий.

Формулы, полученные в работе [4], и уравнения (11)-(16), представленные в настоящей работе, позволяют оценить коэффициенты в разложении эффективного дипольного момента молекул ${ }^{34} \mathrm{~S}^{16} \mathrm{O}_{2},{ }^{32} \mathrm{~S}^{18} \mathrm{O}_{2}$ и ${ }^{32} \mathrm{~S}^{16} \mathrm{O}^{18} \mathrm{O}$. Для реализации данного метода необходимо обладать информацией о значениях равновесного дипольного момента и первых производных дипольного момента по координатам. Однако на настоящий момент в литературе имеется информация о данных величинах только для молекулы ${ }^{32} \mathrm{~S}^{16} \mathrm{O}_{2}[15,16]$. Одним из способов численной оценки параметров дипольного момента изотопологов является применение теории изотопозамещения.

Как было сказано, для любой многоатомной молекулы оператор дипольного момента определяется выражением (6). Учитывая, что дипольный момент в общем случае зависит только от зарядов ядер и геометрии молекулы, можно утверждать, что при изотопозамещении дипольный момент не будет изменяться, т.е. можно записать

$$
\begin{aligned}
P_{z} & =\sum_{\alpha} k_{z \alpha} \mu_{\alpha}=\sum_{\alpha} k_{z \alpha}\left\{\mu_{\alpha}^{e}+\sum_{\lambda} \mu_{\alpha}^{\lambda} Q_{\lambda}\right. \\
& \left.+\sum_{\lambda \leq v} \mu_{\alpha}^{\lambda v} Q_{\lambda} Q_{v} \ldots\right\}=\sum_{\beta} \tilde{k}_{z \beta} \tilde{\mu}_{\beta} \\
& =\sum_{\beta} \tilde{k}_{z \beta}\left\{\tilde{\mu}_{\beta}^{e}+\sum_{\lambda} \tilde{\mu}_{\beta}^{\lambda} \tilde{Q}_{\lambda}+\sum_{\lambda \leq v} \tilde{\mu}_{\beta}^{\lambda \nu} \tilde{Q}_{\lambda} \tilde{Q}_{v} \ldots\right\},
\end{aligned}
$$

где величины с волной относятся к изотопологу. Здесь $Q_{\lambda}=\left(\frac{\hbar}{2 \pi c \omega_{\lambda}}\right) q_{\lambda}$. В случаях одновременного замещения ядер, при котором симметрия молекул не изменяется, справедливо [17] записать равенство

$$
\tilde{k}_{z \beta}=k_{z \beta} \text {. }
$$

Следовательно, равновесный дипольный момент не меняется при таком замещении, т. е. $\mu_{\alpha}^{e}=\tilde{\mu}_{\beta}^{e}$.

Используем общую формулу из работы [17], связывающую нормальные координаты изотополога и „материнской“ молекулы:

$$
Q_{\lambda}=\sum_{\nu} \alpha_{\lambda}^{\nu} \tilde{Q}_{\nu}+\sum_{\mu \nu} \alpha_{\lambda}^{\mu \nu} \tilde{Q}_{\mu} \tilde{Q}_{\nu}+\ldots
$$

В уравнении (19) коэффициенты $\alpha_{\lambda}^{\nu}, \alpha_{\lambda}^{\mu \nu}$ и т. д. определяются из общих формул теории изотопозамещения [17]. Также необходимо отметить, что коэффициенты $\alpha_{\lambda}^{\mu \nu}$ являются „малыми“ по сравнению с коэффициентами $\alpha_{\lambda}^{v}$. В результате подстановки (19) в уравнение (17) несложно получить следующую связь:

$$
\tilde{\mu}_{\beta}^{v}=\sum_{\lambda} \mu_{\beta}^{\lambda} \alpha_{\lambda}^{v}
$$

В то же время при асимметричном замещении $\left({ }^{32} \mathrm{~S}^{16} \mathrm{O}^{18} \mathrm{O} \leftarrow \mathrm{SO}_{2}\right)$, приводящем к понижению симметрии, уравнения (18) и (20) не являются корректными. Введем матрицу, определяющую поворот системы координат, привязанную к центру масс „материнской“ молекулы, приводящую к совпадению с системой координат, привязанной к центру масс изотополога, для любой мгновенной конфигурации ядер. Тогда, согласно работе [17], оператор $\tilde{k}_{z \beta}$ можно переписать как

$$
\tilde{k}_{z \beta}=\sum_{\alpha} k_{z \alpha} K_{\alpha \beta}
$$

где $K_{\alpha \beta}$ является функцией нормальных координат:

$$
K_{\alpha \beta}(Q)=K_{\alpha \beta}^{e}+\sum_{\lambda} K_{\alpha \beta}^{\lambda} Q_{\lambda}+\ldots
$$

Здесь $K_{\alpha \beta}^{e}$ - элементы равновесной матрицы поворота, $K_{\alpha \beta}^{\lambda}$ - коэффициенты, которые могут быть рассчитаны на основе формул теории изотопозамещения [17]. В результате, учитывая переопределение матрицы поворота и (21), уравнение (17) примет вид

$$
\begin{aligned}
& P_{z}=\sum_{\alpha} k_{z \alpha}\left\{\mu_{\alpha}^{e}+\sum_{\lambda} \mu_{\alpha}^{\lambda} Q_{\lambda}+\ldots\right\} \\
& =\sum_{\alpha} \sum_{\beta} k_{z \alpha}\left\{K_{\alpha \beta}^{e}+\sum_{\lambda} K_{\alpha \beta}^{\lambda} Q_{\lambda}+\ldots\right\} \\
& \times\left\{\tilde{\mu}_{\beta}^{e}+\sum_{v} \tilde{\mu}_{\beta}^{v} \tilde{Q}_{v}+\ldots\right\} .
\end{aligned}
$$

Отсюда просто получить, что

$$
\mu_{\alpha}^{e}=\sum_{\beta} K_{\alpha \beta}^{e} \tilde{\mu}_{\beta}^{e}
$$

и

$$
\sum_{\lambda} \mu_{\alpha}^{\lambda} Q_{\lambda}=\sum_{\beta} K_{\alpha \beta}^{e} \sum_{\nu} \tilde{\mu}_{\beta}^{\nu} \tilde{Q}_{\nu}+\sum_{\lambda \beta} K_{\alpha \beta}^{\lambda} \tilde{\mu}_{\beta}^{e} Q_{\lambda} .
$$


Таблица 4. Параметры эффективного дипольного момента молекулы HDO, debye

\begin{tabular}{c|c|c}
\hline \multirow{2}{*}{$\begin{array}{c}\text { Коэффи- } \\
\text { циент }\end{array}$} & {$[21]$} & Расчет \\
\cline { 2 - 3 } & 0.10053 & 0.0931 \\
\hline${ }^{010} \mu_{1}$ & $-5.308 \cdot 10^{-3}$ & $5.816 \cdot 10^{-3}$ \\
\hline${ }^{010} \mu_{4}$ & $-9.648 \cdot 10^{-4}$ & $-1.947 \cdot 10^{-4}$ \\
\hline${ }^{010} \mu_{5}$ & $7.781 \cdot 10^{-2}$ & $6.718 \cdot 10^{-2}$ \\
\hline${ }^{010} \mu_{9}$ & $-2.133 \cdot 10^{-3}$ & $-4.213 \cdot 10^{-4}$ \\
\hline${ }^{010} \mu_{12}$ & $2.633 \cdot 10^{-4}$ & $2.770 \cdot 10^{-4}$ \\
\hline${ }^{010} \mu_{14}$ & &
\end{tabular}

Учитывая необходимое условие, накладываемое на матрицу $K_{\alpha \beta}^{e}$, а именно условие ортогональности $K_{\alpha \beta}^{e} K_{\alpha \gamma}^{e}=\delta_{\beta \gamma}$, имеем

$$
\begin{gathered}
\tilde{\mu}_{\gamma}^{e}=\sum_{\alpha} K_{\alpha \gamma}^{e} \mu_{\alpha}^{e}, \\
\sum_{\nu} \tilde{\mu}_{\gamma}^{v} \tilde{Q}_{\nu}=\sum_{\lambda} \sum_{\alpha} K_{\alpha \gamma}^{e}\left\{\mu_{\alpha}^{\lambda}-\sum_{\beta} K_{\alpha \beta}^{\lambda} \tilde{\mu}_{\beta}^{e}\right\} Q_{\lambda} .
\end{gathered}
$$

С учетом (19) приравняем в уравнении (23) правую и левую части перед координатой $\tilde{Q}_{\nu}$. В результате первая производная дипольного момента изотопозамещенной молекулы определяется следующим образом:

$$
\tilde{\mu}_{\gamma}^{v}=\sum_{\lambda} \sum_{\alpha} K_{\alpha \gamma}^{e}\left\{\mu_{\alpha}^{\lambda}-\sum_{\beta} \tilde{K}_{\alpha \beta}^{\lambda} \tilde{\mu}_{\beta}^{e}\right\} \alpha_{\lambda}^{v},
$$

где $\widetilde{K}_{\alpha \beta}^{\lambda}=K_{\alpha \beta}^{\lambda}\left(\frac{\hbar}{2 \pi c \omega_{\lambda}}\right)$.

Соотношения (22) и (24) будут в дальнейшем использоваться для численных расчетов параметров в разложении эффективного дипольного момента фундаментальных полос диоксида серы и ее изотопологов. На основе формул из работы [17] были рассчитаны элементы матриц $\alpha_{\lambda}^{v}$, элементы равновесной матрицы поворота $K_{\alpha \beta}^{e}$ и коэффициенты $\widetilde{K}_{\alpha \beta}^{\lambda}$ для рассматриваемых молекул.

\section{5. Изотопозамещение $\mathrm{HDO} \leftarrow \mathrm{H}_{2} \mathrm{O}$}

Для того чтобы убедиться в правильности полученных формул (11)-(16), был проведен тестовый расчет на примере замещения в молекуле воды, а именно $\mathrm{HDO} \leftarrow \mathrm{H}_{2} \mathrm{O}$. Выбор дейтерированной модификации воды HDO обусловлен тем, что данная молекула обладает необходимой симметрией $C_{s}$, а также является достаточно хорошо изученной, что позволяет сравнить полученные результаты с имеющимися в литературе данными. Подобный анализ, к сожалению, нельзя провести для молекулы ${ }^{32} \mathrm{~S}^{16} \mathrm{O}^{18} \mathrm{O}$ в связи с отсутствием в литературе экспериментальной информации о параметрах эффективного дипольного момента. Поэтому данные, касающиеся изотопической модификации диоксида серы, можно считать полученными впервые.

Для молекулы $\mathrm{H}_{2} \mathrm{O}$ постоянный дипольный момент $\mu_{x}^{e}=-1.85498(9)$ D был взят из работы [18], параметры $\mu_{x}^{1}=-0.02167 \mathrm{D}, \mu_{x}^{2}=0.1794 \mathrm{D}, \mu_{z}^{3}=0.0971 \mathrm{D}$ были оценены через известные эффективные параметры дипольного момента для фундаментальных полос молекулы воды $\left({ }^{100} \mu_{x 1}=-0.015323 \pm 0.00074 \mathrm{D}\right.$, ${ }^{010} \mu_{x 1}=0.1269 \pm 0.0014 \mathrm{D},{ }^{001} \mu_{z 1}=0.06869 \pm 0.0013 \mathrm{D}$, взятые из работ $[3,19])$ с помощью соотношения

$$
\mu_{\alpha}^{\lambda}={ }^{v_{\lambda}} \mu_{\alpha j} \sqrt{2}
$$

где $v_{\lambda}$ определяет состояние, соответствующее однократному возбуждению одного из трех колебаний $q_{1}, q_{2}$ или $q_{3}$.

На основании формул (22) и (24) для молекулы HDO были рассчитаны значения постоянных дипольных моментов $\mu_{x}^{e}=-1.72875 \mathrm{D}, \mu_{z}^{e}=-0.672577 \mathrm{D}$ и первых производных дипольного момента: $\mu_{x}^{1}=0.0033 \mathrm{D}$, $\mu_{x}^{2}=0.1317 \quad \mathrm{D}, \mu_{x}^{3}=-0.0417 \quad \mathrm{D}, \mu_{z}^{1}=-0.0507 \quad \mathrm{D}$, $\mu_{z}^{2}=0.0950 \mathrm{D}, \mu_{z}^{3}=0.0618$ D. Для вычисления потребовались следующие значения:

$$
\begin{aligned}
K_{x x}^{e} & =K_{z z}^{e}=0.931953 \\
K_{z x}^{e} & =-K_{x z}^{e}=0.362579, K_{y y}^{e}=1 \\
K_{x x}^{1} & =K_{z z}^{1}=-0.260084 \cdot 10^{-5} \\
K_{x z}^{1} & =-K_{z x}^{1}=0.668450 \cdot 10^{-5} \\
K_{x x}^{2} & =K_{z z}^{2}=0.913262 \cdot 10^{-2} \\
K_{x z}^{2} & =-K_{z x}^{2}=-0.234740 \cdot 10^{-1} \\
K_{x x}^{3} & =K_{z z}^{3}=0.465373 \cdot 10^{-3} \\
K_{x z}^{3} & =-K_{z x}^{1}=-0.119617 \cdot 10^{-2}
\end{aligned}
$$

(все величины $K_{\alpha \beta}^{\lambda}-$ безразмерные);

- $\alpha_{1}^{1}=0.548868, \alpha_{2}^{1}=-0.018909$,

$\alpha_{3}^{1}=-0.477716, \alpha_{1}^{2}=0.004165$,

$\alpha_{2}^{2}=0.876216, \alpha_{3}^{2}=-0.001579$,

$\alpha_{1}^{3}=0.677277, \alpha_{2}^{3}=-0.009765$,

$\alpha_{3}^{3}=0.734877$;

- гармонические частоты $\omega_{1}=3825.8716 \mathrm{~cm}^{-1}$, $\omega_{2}=1650.6604 \mathrm{~cm}^{-1}, \quad \omega_{3}=3936.4166 \mathrm{~cm}^{-1} \quad$ для молекулы воды были оценены на основе параметров внутримолекулярной потенциальной функции молекулы $\mathrm{H}_{2} \mathrm{O}$, взятой из работы [20]; гармонические частоты $\tilde{\omega}_{1}=2819.5001 \mathrm{~cm}^{-1}, \tilde{\omega}_{2}=1446.6151 \mathrm{~cm}^{-1}$, $\tilde{\omega}_{3}=3883.6413 \mathrm{~cm}^{-1}$ для молекулы HDO были рассчитаны в соответствии с формулами из работы [17].

На основании вышеизложенного были рассчитаны коэффициенты ${ }^{a_{i}} \mu_{j}$ для $1,4,5,9,12$ и 14 параметров в разложении эффективного дипольного момента 
Таблица 5. Параметры дипольного момента молекулы диоксида серы и его изотопологов, debye

\begin{tabular}{c|c|c|c|c}
\hline Параметры & ${ }^{32} \mathrm{~S}^{16} \mathrm{O}_{2}{ }^{1}$ & ${ }^{34} \mathrm{~S}^{16} \mathrm{O}_{2}$ & ${ }^{32} \mathrm{~S}^{18} \mathrm{O}_{2}$ & ${ }^{32} \mathrm{~S}^{16} \mathrm{O}^{18} \mathrm{O}$ \\
\hline$\mu_{x}^{e}$ & 1.63308 & $1.63308^{1}$ & $1.63308^{1}$ & 1.6327 \\
$\mu_{x}^{1}$ & 0.130 & 0.125 & 0.12931 & 0.130 \\
$\mu_{x}^{2}$ & -0.194 & -0.192 & -0.185 & -0.190 \\
$\mu_{z}^{3}$ & -0.330 & -0.325 & -0.319 & -0.324 \\
$\mu_{z}^{e}$ & 0 & 0 & 0 & -0.033 \\
$\mu_{z}^{1}$ & 0 & 0 & 0 & -0.028 \\
$\mu_{z}^{2}$ & 0 & 0 & 0 & -0.002 \\
$\mu_{x}^{3}$ & 0 & 0 & 0 & -0.008
\end{tabular}

Примечание. ${ }^{1}[15]$.

с использованием полученных в данной работе формул (11)-(16). Результат расчета приведен в табл. 4. Необходимо отметить, что данный анализ был проведен без учета резонансов, так как в литературе отсутствует информация о подобных исследованиях.

Из табл. 4 можно видеть неплохое соответствие между абсолютными значениями, предсказанными на основе соотношений (11)-(16), и экспериментальными данными для 1, 4, 9 и 14 параметров (относительная погрешность составляет 7.9, 8.7, 15.8 и 4.9\% соответственно). Однако имеет место отличие в знаке параметра ${ }^{010} \mu_{4}$, полученного при расчете, в сравнении с экспериментально определенным значением. Такое несоответствие имеет следующее объяснение: из эксперимента возможно определить только абсолютные значения параметров ${ }^{a_{i}} \mu_{j}$, поскольку интенсивность спектральной линии пропорциональна квадрату матричного элемента от дипольного момента (см. уравнение (1)); поэтому остается произвол в определении знаков параметров разложения.

Имеется также существенное различие между расчетными и экспериментальными значениями параметров 5 и 12. Мы полагаем, что это связано с особенностями решения обратной задачи при определении параметров эффективного дипольного момента. Дело в том, что если параметры первого порядка (1 и 9) должны иметь достаточно определенные значения, чтобы воспроизводить интенсивности с экспериментальной точностью, то параметры более высоких порядков, в частности 5 и 12, вносят менее заметный вклад в описание интенсивностей, поскольку имеют больший порядок малости. Таким образом, можно получить несколько наборов параметров, которые одинаково эффективно восстанавливают экспериментальные значения. В дополнение к этому, поскольку центробежные поправки в параметры эффективного дипольного момента определяются из эксперимента, как правило, с невысокой точностью, то даже сильное различие в предсказанных и „экспериментальных“ значениях таких параметров не следует воспринимать как некорректность теоретической модели.

\section{6. Изотопозамещение ${ }^{32} \mathrm{~S}^{16} \mathrm{O}^{18} \mathrm{O} \leftarrow{ }^{32} \mathrm{~S}^{16} \mathrm{O}_{2}$}

При изотопозамещении $\mathrm{XYZ} \leftarrow \mathrm{XY}_{2}$ равновесная система координат претерпевает поворот. Как следствие, появляются две компоненты не только постоянного дипольного момента, но и всех производных дипольного момента по нормальным координатам. Для исследуемого несимметричного замещения ${ }^{32} \mathrm{~S}^{16} \mathrm{O}^{18} \mathrm{O} \leftarrow \mathrm{SO}_{2}$, как показали расчеты, угол поворота системы координат составил около $1^{\circ}$. Как видно из табл. 5, из-за малости угла равновесный дипольный момент $\mu_{x}^{e}$ незначительно изменяется по сравнению с соответствующим параметром „материнской“ молекулы, а параметр $\mu_{z}^{e}-$ компонента равновесного дипольного момента вдоль оси $z-$ принимает отличное от нуля малое значение $(-0.033 \mathrm{D})$. По этой же причине мало меняется производная (в нашем случае первая) оператора дипольного момента по нормальным координатам.

Для расчета параметров дипольного момента изотопологов диоксида серы были использованы формулы (22) и (24). Постоянный дипольный момент $\mu_{x}^{e}=1.63308 \mathrm{D}$, значения первых производных дипольного момента по координатам $\mu_{x}^{1}=0.130 \mathrm{D}$, $\mu_{x}^{2}=-0.194 \mathrm{D}, \mu_{z}^{3}=-0.330 \mathrm{D}$ были взяты из работы [15]. Для вычисления параметров дипольного момента молекулы-изотополога ${ }^{32} \mathrm{~S}^{16} \mathrm{O}^{18} \mathrm{O}(24)$ необходимо использовать элементы матриц $\alpha, K_{e}$ и $K^{\lambda}$. Численно указанные матрицы были получены с помощью формул из работы [17]:

- $K_{x x}^{e}=K_{z z}^{e}=0.999795$, $K_{z x}^{e}=-K_{x z}^{e}=0.020229, K_{y y}^{e}=1$;

- $K_{x x}^{1}=K_{z z}^{1}=0.534705 \cdot 10^{-5}$, $K_{x z}^{1}=-K_{z x}^{1}=-0.264276 \cdot 10^{-3}$, $K_{x x}^{2}=K_{z z}^{2}=-0.267172 \cdot 10^{-4}$, $K_{x z}^{2}=-K_{z x}^{2}=0.1320488 \cdot 10^{-2}$, $K_{x x}^{3}=K_{z z}^{3}=0.346737 \cdot 10^{-5}$, $K_{x z}^{3}=-K_{z x}^{1}=-0.171373 \cdot 10^{-3}$ (все величины $K_{\alpha \beta}^{\lambda}$ являются безразмерными);

- $\alpha_{1}^{1}=0.969174, \alpha_{2}^{1}=-0.019251$, $\alpha_{3}^{1}=0.089965, \alpha_{1}^{2}=0.003631$,

$\alpha_{2}^{2}=0.980094, \alpha_{3}^{2}=0.002024$, $\alpha_{1}^{3}=-0.127410, \alpha_{2}^{3}=-0.011805$, $\alpha_{3}^{3}=0.979813$;

- гармонические частоты $\widetilde{\omega}_{1}=1138.5300 \mathrm{~cm}^{-1}$, $\widetilde{\omega}_{2}=511.8415 \mathrm{~cm}^{-1}, \widetilde{\omega}_{3}=1362.0394 \mathrm{~cm}^{-1}$ для молекулы ${ }^{32} \mathrm{~S}^{16} \mathrm{O}^{18} \mathrm{O}$ были оценены на основе формул изотопозамещения [17], при этом использовались гармонические частоты молекулы ${ }^{32} \mathrm{SO}_{2}$ вычисленые на основе параметров внутримолекулярной потенциальной функции, взятых из работы [22]. 
Таблица 6. Рассчитанные и экспериментальные значения параметров эффективного дипольного момента молекул ${ }^{32} \mathrm{~S}^{16} \mathrm{O}_{2},{ }^{34} \mathrm{~S}^{16} \mathrm{O}_{2}$, ${ }^{32} \mathrm{~S}^{16} \mathrm{O}^{18} \mathrm{O}$, debye

\begin{tabular}{|c|c|c|c|c|c|c|}
\hline Полоса & & \multicolumn{2}{|c|}{${ }^{32} \mathrm{~S}^{16} \mathrm{O}_{2}$} & \multicolumn{2}{|c|}{${ }^{34} \mathrm{~S}^{16} \mathrm{O}_{2}$} & \multirow{2}{*}{$\frac{{ }^{32} \mathrm{~S}^{16} \mathrm{O}^{18} \mathrm{O}}{7}$} \\
\hline 1 & 2 & 3 & 4 & 5 & 6 & \\
\hline \multirow{7}{*}{$v_{1}$} & Параметр & {$[24]$} & Расчет & {$[25]$} & Расчет & Расчет \\
\hline & ${ }^{100} \mu_{1}$ & $0.89774 \cdot 10^{-1}$ & $0.919 \cdot 10^{-1}$ & $0.8796 \cdot 10^{-1}$ & $0.889 \cdot 10^{-1}$ & $0.921 \cdot 10^{-1}$ \\
\hline & ${ }^{100} \mu_{4}$ & $-0.1987 \cdot 10^{-3}$ & $-0.146 \cdot 10^{-3}$ & $-0.17881 \cdot 10^{-3}$ & $-0.139 \cdot 10^{-3}$ & $-0.146 \cdot 10^{-3}$ \\
\hline & ${ }^{100} \mu_{5}$ & $-0.12923 \cdot 10^{-3}$ & $-0.105 \cdot 10^{-3}$ & $-0.9118 \cdot 10^{-4}$ & $-0.103 \cdot 10^{-3}$ & $-0.745 \cdot 10^{-4}$ \\
\hline & ${ }^{100} \mu_{9}$ & 0 & 0 & 0 & 0 & $-0.195 \cdot 10^{-1}$ \\
\hline & ${ }^{100} \mu_{12}$ & 0 & 0 & 0 & 0 & $0.696 \cdot 10^{-5}$ \\
\hline & ${ }^{100} \mu_{14}$ & 0 & 0 & 0 & 0 & $-0.186 \cdot 10^{-5}$ \\
\hline \multirow{7}{*}{$v_{2}$} & Параметр & {$[26]$} & Расчет & {$[27]$} & Расчет & Расчет \\
\hline & ${ }^{010} \mu_{1}$ & -0.13573 & -0.1372 & -0.14802 & -0.136 & -0.134 \\
\hline & ${ }^{010} \mu_{4}$ & $0.585 \cdot 10^{-3}$ & $0.598 \cdot 10^{-3}$ & $0.5694 \cdot 10^{-3}$ & $0.585 \cdot 10^{-3}$ & $0.586 \cdot 10^{-3}$ \\
\hline & ${ }^{010} \mu_{5}$ & $0.533 \cdot 10^{-4}$ & $0.559 \cdot 10^{-4}$ & $0.533 \cdot 10^{-4}$ & $0.568 \cdot 10^{-4}$ & $0.769 \cdot 10^{-4}$ \\
\hline & ${ }^{010} \mu_{9}$ & 0 & 0 & 0 & 0 & $-0.169 \cdot 10^{-2}$ \\
\hline & ${ }^{010} \mu_{12}$ & 0 & 0 & 0 & 0 & $-0.103 \cdot 10^{-6}$ \\
\hline & ${ }^{010} \mu_{14}$ & 0 & 0 & 0 & 0 & $-0.311 \cdot 10^{-5}$ \\
\hline \multirow{7}{*}{$v_{3}$} & Параметр & {$[24]$} & Расчет & {$[25]$} & Расчет & Расчет \\
\hline & ${ }^{001} \mu_{1}$ & 0 & 0 & 0 & 0 & $-0.546 \cdot 10^{-2}$ \\
\hline & ${ }^{001} \mu_{4}$ & 0 & 0 & 0 & 0 & $0.105 \cdot 10^{-4}$ \\
\hline & ${ }^{001} \mu_{5}$ & 0 & 0 & 0 & 0 & $-0.773 \cdot 10^{-5}$ \\
\hline & ${ }^{001} \mu_{9}$ & -0.226667 & -0.233 & -0.22508 & -0.230 & -0.229 \\
\hline & ${ }^{001} \mu_{12}$ & $-0.182 \cdot 10^{-3}$ & $0.131 \cdot 10^{-3}$ & $0.979 \cdot 10^{-4}$ & $0.131 \cdot 10^{-3}$ & $0.992 \cdot 10^{-4}$ \\
\hline & ${ }^{001} \mu_{14}$ & $0.742 \cdot 10^{-4}$ & $0.733 \cdot 10^{-4}$ & $0.700 \cdot 10^{-4}$ & $0.738 \cdot 10^{-4}$ & $0.450 \cdot 10^{-4}$ \\
\hline
\end{tabular}

Формулы (11)-(16) позволили численно определить для молекулы ${ }^{32} \mathrm{~S}^{16} \mathrm{O}^{18} \mathrm{O}$ шесть наиболее значимых параметров эффективного дипольного момента (табл. 6, колонка 7). При этом теоретические значения параметров эффективного дипольного момента для молекулы ${ }^{32} \mathrm{~S}^{16} \mathrm{O}^{18} \mathrm{O}$ мы не имеем возможности сравнить с экспериментальными, но качественно они хорошо характеризуют общую тенденцию соотношения интенсивностей переходов $a$ - и $b$-типов в гибридных фундаментальных полосах $v_{1}$ и $v_{3}$ [23].

Данные параметры получены впервые и могут быть использованы в качестве начального приближения в процессе определения интенсивностей колебательновращательных линий фундаментальных полос молекулы ${ }^{32} \mathrm{~S}^{16} \mathrm{O}^{18} \mathrm{O}$. Следует учесть, что анализ интенсивностей линий для этого изотополога является наиболее сложным, так как получить данную молекулу в чистом виде (с содержанием в образце 100\%) довольно трудно, поскольку она является неустойчивой и легко перехо- дит в другие модификации. В этом свете выполненное исследование представляется весьма актуальным.

\section{7. Заключение}

Применительно к молекуле диоксида серы и ее изотопологам были рассчитаны параметры дипольного момента (табл. 5) и эффективного дипольного момента, численные значения которых приведены в табл. 6 (колонки 4, 6 и 7). Для случаев, когда замещение ядер не изменяет симметрию молекулы-изотополога, влияние изотопозамещения слабое, и как следствие, параметры изотопологов мало изменяются относительно соответствующих параметров „материнской“ молекулы (табл. 5 и 6 для молекул ${ }^{32} \mathrm{~S}^{16} \mathrm{O}_{2},{ }^{34} \mathrm{~S}^{16} \mathrm{O}_{2},{ }^{32} \mathrm{~S}^{18} \mathrm{O}_{2}$ ). При несимметричном замещении $\left({ }^{32} \mathrm{~S}^{16} \mathrm{O}^{18} \mathrm{O} \leftarrow{ }^{32} \mathrm{SO}_{2}\right)$ влияние изотопозамещения проявляется также слабо. Однако в отличие от молекул типа $\mathrm{XY}_{2} \quad\left(C_{2 v}\right.$ симметрия), для которых используется набор из восьми параметров 
эффективного дипольного момента, для описания интенсивностей колебательно-вращательных линий молекул типа $\mathrm{XYZ}\left(\right.$ симметрия $C_{s}$ ) необходимо 16 параметров эффективного дипольного момента. Следовательно, при исследовании несимметрично замещенных изотопологов диоксида серы физически разумные параметры эффективного дипольного момента должны отличаться от соответствующих параметров основного изотополога не более, чем на несколько процентов. При этом параметры ${ }^{\tau_{i}} \mu_{9}, \tau_{i} \mu_{12}$ и ${ }^{\tau_{i}} \mu_{14}$ вносят существенный вклад в интенсивность линий и поэтому должны принимать не равные нулю значения. Полученные результаты вполне характеризуют вышеописанную тенденцию. Как можно видеть из табл. 6 (колонки 3 и 7), изменение параметров эффективного дипольного момента молекулы ${ }^{32} \mathrm{~S}^{16} \mathrm{O}^{18} \mathrm{O}$ относительно соответствующих параметров молекулы ${ }^{32} \mathrm{SO}_{2}$ является незначительным, при этом параметры, отвечающие за переходы типа $a$ - в случае $\tau_{i}=(100)$, (010) $\left({ }^{\tau_{i}} \mu_{9},{ }^{\tau_{i}} \mu_{12}\right.$ и $\left.{ }^{\tau_{i}} \mu_{14}\right)$ и $b$-, когда $\tau_{i}=(001)\left({ }^{\tau_{i}} \mu_{1},{ }^{\tau_{i}} \mu_{4}\right.$ и $\left.\tau_{i} \mu_{5}\right)$ не равны нулю.

Полученные выражения могут быть использованы в общем случае для молекул, в которых влияние изотопозамещения существенно и возникает необходимость теоретически предсказывать значения параметров эффективного дипольного момента.

\section{Финансирование работы}

Работа выполнена при поддержке Российского фонда фундаментальных исследований (проект № 18-32-00116 мол_а).

\section{Конфликт интересов}

Авторы заявляют, что у них нет конфликта интересов.

\section{Список литературы}

[1] Self S., Rampino M.R., Barbera J.J. // J. Volcanol. Geotherm. Res. 1981. V. 11. P. 41-60. doi 10.1016/0377-0273(81)90074-3

[2] McCormic M.P., Thomason L.W., Trepte C.R. // Nature. 1995. V. 373. N 6513. P. 99-404. doi $10.1038 / 373399 \mathrm{a} 0$

[3] Flaud J.M., Camy-Peyret C. // J. Mol. Spectr. 1975. V. 55. P. 278-310. doi 10.1016/0022-2852(75)90270-2

[4] Camy-Peyret C., Flaud J.M. Molecular Spectroscopy: Modern Research. Volume III. Orlando: Academic press, 1985. 452 p.

[5] Sarka K., Danielache S.O., Kondorskiy A., Nanbu S. // Chem. Phys. 2019. V. 516. P. 108-115. doi 10.1016/j.chemphys.2018.08.045

[6] Karlovets E.V., Perevalov V.I. // Opt. Spectr. 2015. V. 119. N 1. P. 16-21. doi 10.1134/S0030400X15070139

[7] Gangemi F.A.// J. Chem. Phys. 1963. V. 39. N 12. P. 3490-3496. doi 10.1063/1.1734218

[8] Perevalov V.I., Lobodenko E.I., Lyulin O.M., Teffo J.L. // J. Mol. Spectr. 1995. V. 171. N 2. P. 435-452. doi 10.1006/jmsp.1995.1131

[9] Watson J.K.G. // Mol. Phys. 1968. V. 15. N 5. P. 479-490. doi 10.1080/00268976800101381
[10] Papousek D., Aliev M.R. Molecular Vibration-Rotation Spectra. Amsterdam, Oxford, N.Y.: Elsevier, 1982. 324 p.

[11] Nielsen H.H. // Rev. Mod. Phys. 1951. V. 23. P. 90-136. doi 10.1103/RevModPhys.23.90

[12] Howard B.J., Moss R.E. // Mol. Phys. 1970. V. 19. P. 433-450. doi 10.1080/00268977000101471

[13] Jorgensen F., Pedersen T. // Mol. Phys. 1974. V. 27. N 1. P. 33-47. doi 10.1080/00268977400100041

[14] Ulenikov O.N., Tochenov R.N., Qing-Shi Zhu // Spectr. Acta A. 1996. V. 52. P. 1829-1841. doi 10.1016/S0584-8539(96)01749-7

[15] Patel D., Margolese D., Dyke T.R. // J. Chem. Phys. 1979. V. 70. N 6. P. 2740-2747. doi 10.1063/1.437860

[16] Secroun C., Barbe A., Jouve P. // J. Mol. Spectr. 1973. V. 45. P. 1-9. doi 10.1016/0022-2852(73)90170-7

[17] Bykov A.D., Makushkin Yu.S., Ulenikov O.N. // J. Mol. Spectr. 1981. V. 85. P. 462-479. doi 10.1016/0022-2852(81)90217-4

[18] Shostak S.S., Ebenstein W.L., Muenter J.S. // J. Chem. Phys. 1991. V. 94. N 9. P. 5875-5882. doi 10.1063/1.460471

[19] Camy-Peyret C., Flaud J.M. // Mol. Phys. 1976. V. 32. P. 499-521. doi 10.1080/00268977600103251

[20] Halonen L., Carrington Jr.T. // J. Chem. Phys. 1988. V. 88. N 7. P. 4171-4185. doi 10.1063/1.453824

[21] Toth R.A. // J. Mol. Spectr. 1993. V. 162. P. 20. doi 10.1006/jmsp.1993.1266

[22] Ulenikov O.N., Bekhtereva E.S., Alanko S., Horneman V.-M., Gromova O.V., Leroy C. // J. Mol. Spectr. 2009. V. 257. P. 137-156. doi 10.1016/j.jms.2009.07.005

[23] Ulenikov O.N., Bekhtereva E.S., Krivchikova Y., Zamotaeva V.A., Buttersack T., Sydow C., Bauerecker S. // J. Quant. Spectrosc. Radiat. Transfer. 2016. V. 168. P. 29-39. doi 10.1016/j.jqsrt.2015.08.010

[24] Chu P.M., Wetzel S.J., Lafferty W.J., Perrin A., Flaud J.M., Arcas Ph., Guelachvili G. // J. Mol.Spectr. 1998. V. 189. P. 55-63. doi 10.1006/jmsp.1997.7517

[25] Flaud J.-M., Lafferty W.J., Sams R.L. // J. Quant. Spectrosc. Radiat. Transfer. 2009. V. 110. P. 669-674. doi 10.1016/j.jqsrt.2008.12.003

[26] Ulenikov O.N., Bekhtereva E.S., Gromova O.V., Quack M., Mellau G.C., Sydow C., Bauerecker S. // J. Quant. Spectrosc. Radiat. Transfer. 2018. V. 210. P. 141-155. doi 10.1016/j.jqsrt.2018.02.010

[27] Ulenikov O.N., Bekhtereva E.S., Gromova O.V., Ziatkova A.G., Quack M., Mellau G.Ch., Sydow C., Bauerecker S. // J. Quant. Spectrosc. Radiat. Transfer. 2019. V. 229. P. 166-178. doi $10.1016 /$ j.jqsrt.2018.11.031 